\title{
Methylation-associated down-regulation of RASSF1A and up-regulation of RASSF1C in pancreatic endocrine tumors
}

\author{
Giorgio Malpeli ${ }^{1 * \dagger}$, Eliana Amato ${ }^{2 *}$, Mario Dandrea ${ }^{1}$, Caterina Fumagalli ${ }^{3}$, Valentina Debattisti ${ }^{1}$, Letizia Boninsegna ${ }^{4}$,
} Giuseppe Pelosi ${ }^{3}$, Massimo Falconi ${ }^{4}$ and Aldo Scarpa ${ }^{1,2}$

\begin{abstract}
Background: RASSFIA gene silencing by DNA methylation has been suggested as a major event in pancreatic endocrine tumor (PET) but RASSF1A expression has never been studied. The RASSF1 locus contains two CpG islands ( $A$ and $C$ ) and generates seven transcripts (RASSF1A-RASSF1G) by differential promoter usage and alternative splicing.

Methods: We studied 20 primary PETs, their matched normal pancreas and three PET cell lines for the (i) methylation status of the RASSF1 CpG islands using methylation-specific PCR and pyrosequencing and (ii) expression of RASSF1 isoforms by quantitative RT-PCR in 13 cases. CpG island A methylation was evaluated by methylation-specific PCR (MSP) and by quantitative methylation-specific PCR (qMSP); pyrosequencing was applied to quantify the methylation of 51 CpGs also encompassing those explored by MSP and qMSP approaches.

Results: MSP detected methylation in 16/20 (80\%) PETs and 13/20 (65\%) normal pancreas. At qMSP, 11/20 PETs (55\%) and 9/20 (45\%) normals were methylated in at least 20\% of RASSF1A alleles.

Pyrosequencing showed variable distribution and levels of methylation within and among samples, with PETs having average methylation higher than normals in $15 / 20(75 \%)$ cases $(P=0.01)$. The evaluation of mRNA expression of RASSF1 variants showed that: i) RASSF1A was always expressed in PET and normal tissues, but it was, on average, expressed 6.8 times less in PET ( $P=0.003)$; ii) RASSF1A methylation inversely correlated with its expression; iii) RASSF1 isoforms were rarely found, except for RASSF1B that was always expressed and RASSF1C whose expression was 11.4 times higher in PET than in normal tissue $(P=0.001)$. A correlation between RASSF1A expression and gene methylation was found in two of the three PET cell lines, which also showed a significant increase in RASSF1A expression upon demethylating treatment.
\end{abstract}

Conclusions: RASSFIA gene methylation in PET is higher than normal pancreas in no more than $75 \%$ of cases and as such it cannot be considered a marker for this neoplasm. RASSF1A is always expressed in PET and normal pancreas and its levels are inversely correlated with gene methylation. Isoform RASSF1C is overexpressed in PET and the recent demonstration of its involvement in the regulation of the Wht pathway points to a potential pathogenetic role in tumor development.

\footnotetext{
*Correspondence: giorgio.malpeli@univr.it; eliana.amato@univr.it

† Contributed equally

${ }^{1}$ Department of Pathology and Diagnostics, University of Verona, Verona,

Italy

${ }^{2}$ ARC-NET Center for Applied Research on Cancer, the Hospital and

University of Verona, Verona, Italy

Full list of author information is available at the end of the article
}

\section{Ciomed Central}

(c) 2011 Malpeli et al; licensee BioMed Central Ltd. This is an Open Access article distributed under the terms of the Creative Commons Attribution License (http://creativecommons.org/licenses/by/2.0), which permits unrestricted use, distribution, and reproduction in any medium, provided the original work is properly cited. 


\section{Background}

Pancreatic endocrine tumors (PET) are rare neoplasms whose molecular pathogenesis is largely unknown. Silencing of the RASSF1A gene by methylation has been proposed as a crucial pathogenetic event in PET by five studies, all of which used the very same methylationspecific PCR (MSP) assay to interrogate the same region of the gene [1-5]. Two of these five papers assessed the methylation status of several candidate tumor suppressor genes and reported the methylation of RASSF1A in $75 \%$ [3] and $83 \%$ [2] of PET. This high rate of RASSF1A methylation in PETs was confirmed in the other three studies, where the rate reported ranged from $60 \%$ to $100 \%$ of cases $[1,4,5]$. However, the formal proof that the RASSF1A gene silencing by methylation in PET is associated with loss of its expression has never been reported.

In tumor types other than PET, RASSF1A involvement was assessed by a quantitative MSP assay (qMSP), analyzing a region of $R A S S F 1 A$ different from the one investigated by MSP in PET [6-10]. For some of these tumors methylation was associated with down-regulation of the gene expression [11-19].

Ras Association Domain Family 1 (RASSF1) is a putative tumor suppressor gene localized at chromosome 3 p21.3 that has been reported to inhibit tumor growth in in vitro and in vivo systems [20-23]. RASSF1 locus generates seven different transcript variants (RASSF1A$G)$ by differential promoter usage and alternative splicing [24]. RASSF1A has been considered a player of inhibitory functions of Ras on cell growth by acting as downstream agent and as such its inactivation is believed to result in the indirect activation of the Ras pathway. Other studies however have also assigned complex roles in cell functions to this gene $[25,26]$.

Two CpG islands are associated with RASSF1 regulatory region: $\mathrm{CpG}$ island A extending in the regulatory region common to $R A S S F 1 A, D, E, F$ and $G$; CpG island $\mathrm{C}$ in the regulatory region of $R A S S F 1 C$. Little is known about the influence of CpG islands on RASSF1B. The main RASSF1 variants that are ubiquitously expressed in normal tissues are RASSF $1 A$ and RASSF $1 C$ [24]. No information is available regarding the expression of RASSF1 isoforms in PET.

In this study, we evaluated the putative role of RASSF1A methylation as a possible tumor specific and transcription regulatory event occurring in PET. To this end, we applied both the MSP and qMSP approaches indicated above in a set of 20 PETs and matched normal pancreas together with the evaluation of RASSF1 gene expression in 13 cases. We also assessed the methylation status of each of the $\mathrm{CpG}$ sites encompassing a large area of the regulatory region, including the CpGs interrogated by MSP and qMSP assays, with methylation-sensitive pyrosequencing. This technique allows relative quantification of methylated $\mathrm{CpGs}$ in relation to unmethylated CpGs for each of the CpG sites tested.

\section{Methods}

\section{Samples and nucleic acids extraction}

Twenty primary sporadic PET (Table 1) with matched normal pancreas were studied. Samples were obtained in accordance with the Verona University and Hospital Trust Ethics Committee and PET were classified according to WHO criteria [27]. Normal pancreas was taken far away (at least $2 \mathrm{~cm}$ ) from the neoplastic lesion and was macroscopically and histologically normal at pathological evaluation. All PET samples were negative for MEN1 gene mutation (data not shown) and there was no family history for $M E N 1, V H L$ or any other familial cancer predisposing syndrome.

Nucleic acids were prepared from 12 cryostatic sections, with tumor cellularity checked every four sections. Tumor cellularity was always over $90 \%$. DNA was purified by QiAamp DNA Mini Kit (Qiagen), following manufacturer's instructions. RNA was extracted with TRIzol Reagent (Invitrogen) following the manufacturer instructions, then treated with DNAse I (Invitrogen) for 15 minutes at room temperature, and finally incubated at $65^{\circ} \mathrm{C}$ for 10 minutes for enzyme inactivation. As a control of the DNAse I efficiency, a DNA sample was

\section{Table 1 Clinical data of PET cases}

\begin{tabular}{|c|c|c|c|c|}
\hline Case & Sex & Age (years) & Tumor type & WHO classification* \\
\hline 1 & $M$ & 53 & non-functioning & WDEC \\
\hline 2 & $M$ & 42 & non-functioning & WDEC \\
\hline 3 & M & 51 & insulinoma & WDET \\
\hline 4 & $M$ & 44 & non-functioning & WDEC \\
\hline 5 & $M$ & 48 & non-functioning & WDEC \\
\hline 6 & $M$ & 51 & insulinoma & WDET \\
\hline 7 & $\mathrm{~F}$ & 66 & non-functioning & WDET \\
\hline 8 & $\mathrm{~F}$ & 65 & non-functioning & WDET \\
\hline 9 & $M$ & 35 & non-functioning & WDEC \\
\hline 10 & $\mathrm{~F}$ & 70 & non-functioning & WDEC \\
\hline 11 & $M$ & 76 & non-functioning & WDEC \\
\hline 12 & $\mathrm{~F}$ & 40 & non-functioning & WDEC \\
\hline 13 & $M$ & 41 & insulinoma & WDET \\
\hline 14 & $M$ & 40 & non-functioning & WDEC \\
\hline 15 & $M$ & 68 & non-functioning & WDEC \\
\hline 16 & $\mathrm{~F}$ & 47 & non-functioning & WDET \\
\hline 17 & $M$ & 48 & non-functioning & WDET \\
\hline 18 & $M$ & 41 & gastrinoma & WDEC \\
\hline 19 & $M$ & 70 & non-functioning & WDEC \\
\hline 20 & $\mathrm{~F}$ & 46 & gastrinoma & WDET \\
\hline
\end{tabular}

* WDEC: well-differentiated endocrine carcinoma; WDET: well-differentiated endocrine tumor. 
subjected to DNAse I treatment at the same conditions and loaded on agarose gel.

\section{Cell lines and demethylating treatment}

PET cell lines CM, QGP1 and BON were grown in culture medium containing RPMI 1640 supplemented with $2 \mathrm{mM}$ glutamine and 10\% FBS and incubated at standard conditions $\left(37^{\circ} \mathrm{C}\right.$ with $\left.5 \% \mathrm{CO}_{2}\right)$. Cell lines were treated with the enzymatic inhibitor 5'-aza-2'-deoxycytidine (DAC) (Sigma-Aldrich), previously solubilized in DMSO and stored at $-80^{\circ} \mathrm{C}$ until use. Cells treated with the inhibitor were grown in presence of $2.5 \mu \mathrm{M}$ DAC for 6 days.

\section{Optimization of bisulfite treatment of DNA}

DNA was chemically modified with sodium bisulfite using MethylSeq Kit (Applied Biosystems) to convert unmethylated cytosine to uracil, while methylated cytosines resist conversion. Duration of bisulfite treatment necessary to determine complete cytosines modification was assessed by incubating DNA with sodium bisulfite for 4,8 and 16 hours at $50^{\circ} \mathrm{C}$ in a heat-block. Complete conversion of cytosines occurred after 16 hours treatment and the DNA obtained was used for subsequent experimental procedures.

\section{RASSF1A methylation-specific PCR (MSP)}

Methylation-specific PCR (MSP) was performed according to Pizzi et al. [5]. Reference unmethylated DNA was from healthy donor peripheral blood mononuclear cells. Reference full methylated DNA was CpGenome Universal Methylated DNA (Chemicon International).

\section{Quantitative methylation-specific PCR}

Quantitative MSP (qMSP) was performed as described $[6,8]$ with minor modifications reported in Additional file 1. Primers are listed in Additional file 2, Table S1. The methylation level of RASSF1A was calculated as the ratio of methylated $R A S S F 1 A$ to $M Y O D 1$, the latter representing the total input unmethylated DNA [28]. To classify samples as methylated, we applied the same cutoff used by Xing et al. [10]. The $20 \%$ cut-off point at qMSP analysis was chosen with a conservative assumption that all of the cells in a sample were tumor cells without normal cell contamination and without loss of heterozygosity in the RASSF1A locus. Thus, a cut-off point of $20 \%$ of total alleles for RASSF1A methylation implies that $20 \%$ of all of the tumor cells carry RASSF $1 A$ methylation, if both alleles are methylated in one cell. In reality, the tumor tissue is not $100 \%$ pure, and there may be also loss of heterozygosity in the RASSF $1 A$ locus. Therefore the $20 \%$ cut-off point of total alleles for RASSF1A methylation actually reflects the presence of a clone of more than $20 \%$ of the total number of tumor cells [10].

\section{Pyrosequencing of bisulfite-modified DNA}

Bisulfite-modified DNAs were evaluated by pyrosequencing [29] as recommended (Biotage $A B$ ), using primers and conditions as reported in Additional file 1 and in Additional file 2, Table S1. The degree of methylation at each CpG position was determined from the ratio of $\mathrm{C}$ and $\mathrm{T}$ by the Pyro Q-CpG Software (Biotage AB). Pyrosequencing was performed on the sense and antisense strand of RASSF1A (nucleotides -163 to +262 in chromosome 3: 50353109-50353534, NC_0000003.10) and of RASSF1C (nucleotides -86 to +193 in chromosome 3 : 50349706-50349985, NC_0000003.10).

\section{Identification of alternatively spliced mRNA isoforms of RASSF1 transcribed from CpG island A}

Isoforms $A, D, E, F$ and $G$ originate from alternative splicing of RASSF1A gene. Taking advantage of their different length, isoforms $A, D, E$ and $F$ were identified by microfluidic chip-electrophoretic separation (DNA 1000 chip, 2100 Bioanalyzer, Agilent Technologies) of PCR products. The PCR amplification of cDNAs used primers designed in the first and last exon of the gene (NCBI Reference Sequence NM_007182). As RASSF1G lacks the last exon of RASSF1A, it was amplified separately with opportune primers. The PCR primers used are listed in Additional file 2, Table S1.

\section{Measurement of mRNA expression by quantitative} reverse transcription-polymerase chain reaction (qRT-PCR) RNA samples were retrotranscribed to cDNA using the First Strand cDNA Synthesis Kit (Roche). A reverse transcriptase minus cDNA was prepared for each sample as a control. qRT-PCR conditions and primers are reported in Additional file 1 and in Additional file 2, Table S1. The relative expression level was calculated using transcript level of RPLPO as reference gene and the standard $(=1)$ was the average of the levels of expression of all samples. qRT-PCR data analysis was performed according to the comparative method following the User Bulletin \#2 (Applied Biosystems).

\section{Statistical analysis}

Pearson's correlation ( $r$ ) and Student's $t$-test were used to compare mRNA expression and differentially methylated regions between groups of samples. Pairwise $t$-test or Spearman Rank (rho) correlation was used to compare expression in matched PET and normal samples. $P$ values less than 0.05 were considered statistically significant. 


\section{Results}

The methylation status of $\mathrm{CpG}$ islands $\mathrm{A}$ and $\mathrm{C}$ of the RASSF1 locus (Figure 1) was assessed in a series of 20 PET and matched normal tissues (Table 1). Methylation of $\mathrm{CpG}$ island A was first evaluated by the qualitative methylation-specific PCR (MSP) used in papers dealing with PET [1-5] and then by a quantitative methylation - specific PCR (qMSP) applied in the study of other tumor types [6-10]. The regions of CpG island A analyzed by MSP and qMSP were further investigated by pyrosequencing, which provided the methylation status of $51 \mathrm{CpGs}$ of the island A that also encompasses those explored by MSP and qMSP approaches. Analysis of the expression of the diverse RASSF1 isoforms completed the study. The analysis of CpG island $C$ was performed by pyrosequencing analysis of 37 individual CpGs.

\section{Methylation-specific PCR showed a high frequency of RASSF1A methylation in PET and normal pancreas} MSP detected methylation in 16 of 20 (80\%) PETs and 13 of $20(65 \%)$ normal pancreas (Additional file 3, Figure S1A). In particular, methylation was detected in 12 PETs and matched normals, was absent in both PET and matched normal in 3 cases, while in five cases it was discordantly present in either PET (4 cases) or normal (1 case).

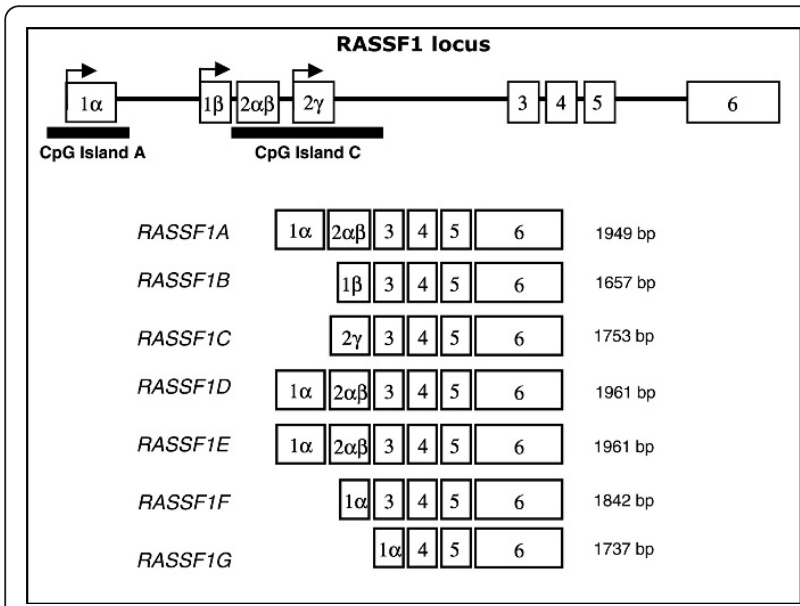

Figure 1 Schematic representation of the RASSF 1 locus on chromosome 3p21.3 and its transcription map. White boxes represent the exons and the bold line represents the introns. RASSF1A, RASSF1B and RASSF1C variants are generated by differential promoter usage (arrows). RASSFID, E, F, G are variants derived from alternative splicings of RASSF1A. Two CpG islands (black bands below the sequence) are associated to RASSF1 promoter region: CpG island A (737 bp, 85 CpGs) extending in the promoter region of RASSF1A, D, E, F and G; CpG island C (1365 bp, 139 CpGs) in the regulatory region of $R A S S F I B$ and $C$.
Quantitative methylation-specific PCR showed variable RASSF1A methylation among PET and normal pancreas All PET and normal samples showed variable levels of methylation at qMSP, but only 11/20 PETs (55\%) and 9/ $20(45 \%)$ normals had a methylation level in at least $20 \%$ of the RASSF1A alleles (Additional file 3, Figure S1B). The cut-off point of $20 \%$ of total alleles for RASSF1A methylation implies that $20 \%$ of cells in a sample carry RASSF1A methylation [10].

Using this cut-off, methylation in paired PET/normal cases was as follows: 6 both methylated, 6 both unmethylated, 5 methylated in PET, 3 methylated in normal. The qMSP methylation levels in PET and matched normals showed a higher degree of methylation in neoplastic tissues with respect to normals in 8 cases and a lower degree in 9 cases; the remaining 3 cases had similar methylation levels in PET and normal.

Pyrosequencing revealed that RASSF1A promoter and first exon are variably methylated within and among PETs and normal pancreas

Pyrosequencing was applied to determine the methylation level of $51 \mathrm{CpGs}$ within $\mathrm{CpG}$ island $\mathrm{A}$ of RASSF1A, including $17 \mathrm{CpGs}$ in the promoter and 34 CpGs in the first exon. In fact, this technique is able to provide the level of methylation of single CpGs (Figure 2). RASSF1A methylation showed a high variability in terms of distribution and level among PET and normal samples (Figure 3). Analysis of the RASSF1A antisense DNA strand confirmed the results obtained for the sense strand.

Table 2 lists the average methylation levels of promoter, first exon and of the CpG island A of RASSF1A in 20 tumors and their matched normal samples. By considering the average methylation of all 51 CpGs, methylation was higher in tumor than in normal tissue in 15/ $20(75 \%)$ cases (pairwise $t$-test, $P=0.01$ ) (Figure 3, Table 2); of the remaining 5 cases, 2 cases had similar methylation level in normal and tumor (cases 1 and 9), while 3 cases had normal showing higher methylation than tumor (cases 2, 6, 16).

By considering a threshold as for qMSP, a sample was defined as "methylated" if it had an average methylation across $51 \mathrm{CpGs}$ higher than 20\%. Using this threshold, $13 / 20(65 \%)$ tumors and 12/20 (60\%) normal samples were methylated. Among the 3 normal samples with higher degree of methylation than the neoplastic counterpart, case 2 showed the largest difference of average methylation level between PET and normal.

Pyrosequencing results overlapped those obtained by qMSP in both PET and normal pancreas when the status of the $10 \mathrm{CpGs}$ included in the qMSP assay (from CpG3 to CpG12; see Figure 3) was considered; in fact, the average methylation level of these $10 \mathrm{CpGs}$ by 


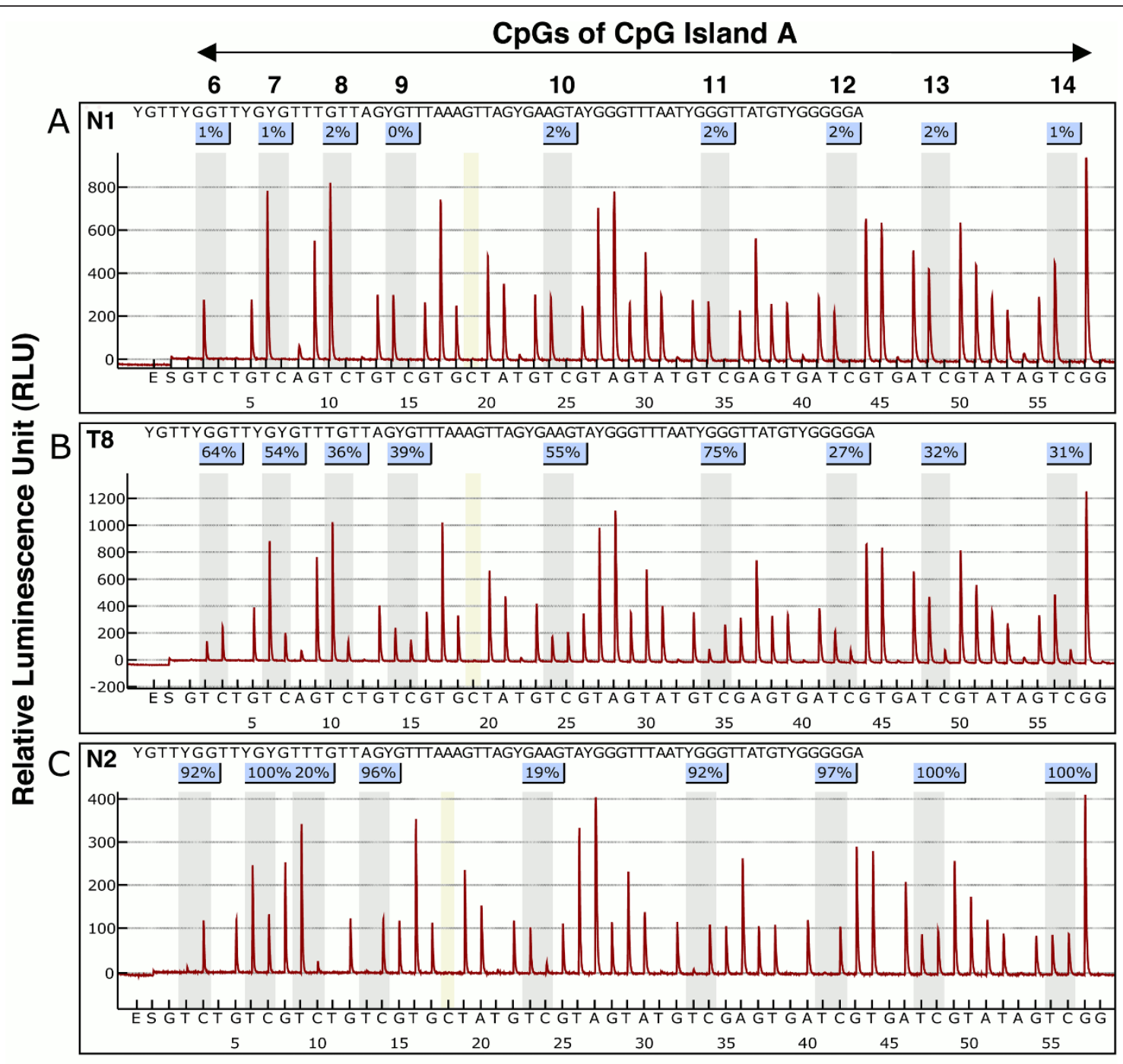

Nucleotide Dispensation Order

Figure 2 Analysis of DNA methylation by pyrosequencing. Representative results of pyrosequencing showing analysis of 9 CpGs in the first exon of RASSF1A (from CpG6 to CpG14). The $y$-axis represents the signal intensity of luminescence in relative luminescence units (RLU) emitted following nucleotide base incorporation into the sequence, while the $x$-axis shows the nucleotide dispensation order, indicated by the letters below each graph: $E$, enzyme mix; $S$, substrate; $A, G, C, T$, nucleotides. The sequence in the upper left of the boxes is the region subjected to pyrosequencing analysis, where the nine letters $Y$ indicate the respective cytosine in the 9 CpG sites (from CpG6 to CpG14) whose methylation status is shown here. Values in light blue boxes are the percentages of methylation of each CpG. Percentages of methylation represent the ratio between signal intensities of $\mathrm{C}$ and $\mathrm{T}$ in each $\mathrm{C}$ of a $\mathrm{CpG}$ site. Dispensations corresponding to the potentially methylated cytosine (C or $T$ after bisulfite treatment) are highlighted in grey. Each panel depicts a representative sample showing no methylation (panel A, sample N1), partial methylation (panel B, sample T8) and high methylation (panel C, sample N2) levels.

pyrosequencing $v s$ the level of methylation by qMSP showed a good correlation $(\mathrm{r}=0.78, P=0.0001)$.

\section{RASSF1A is methylated in normal pancreas}

Regardless of technique used, our data indicate that RASSF1A methylation occurs in normal pancreas, as previously reported by others $[2,4,5,30]$, and presents interindividual variability in terms of frequency and level.
RASSF1A mRNA expression in PET is lower than that of matched normal pancreas and inversely correlates with methylation level of $\mathrm{CpG}$ island $\mathrm{A}$

Expression of RASSF1A mRNA was assessed in 13 PETs and matched normal pancreas by qRT-PCR. Expression was lower in PET than in normal in 11 of 13 cases, equal to an average difference of 6.8 fold $(P=0.003)$ (Figure 4A and Additional file 2, Table S2). 


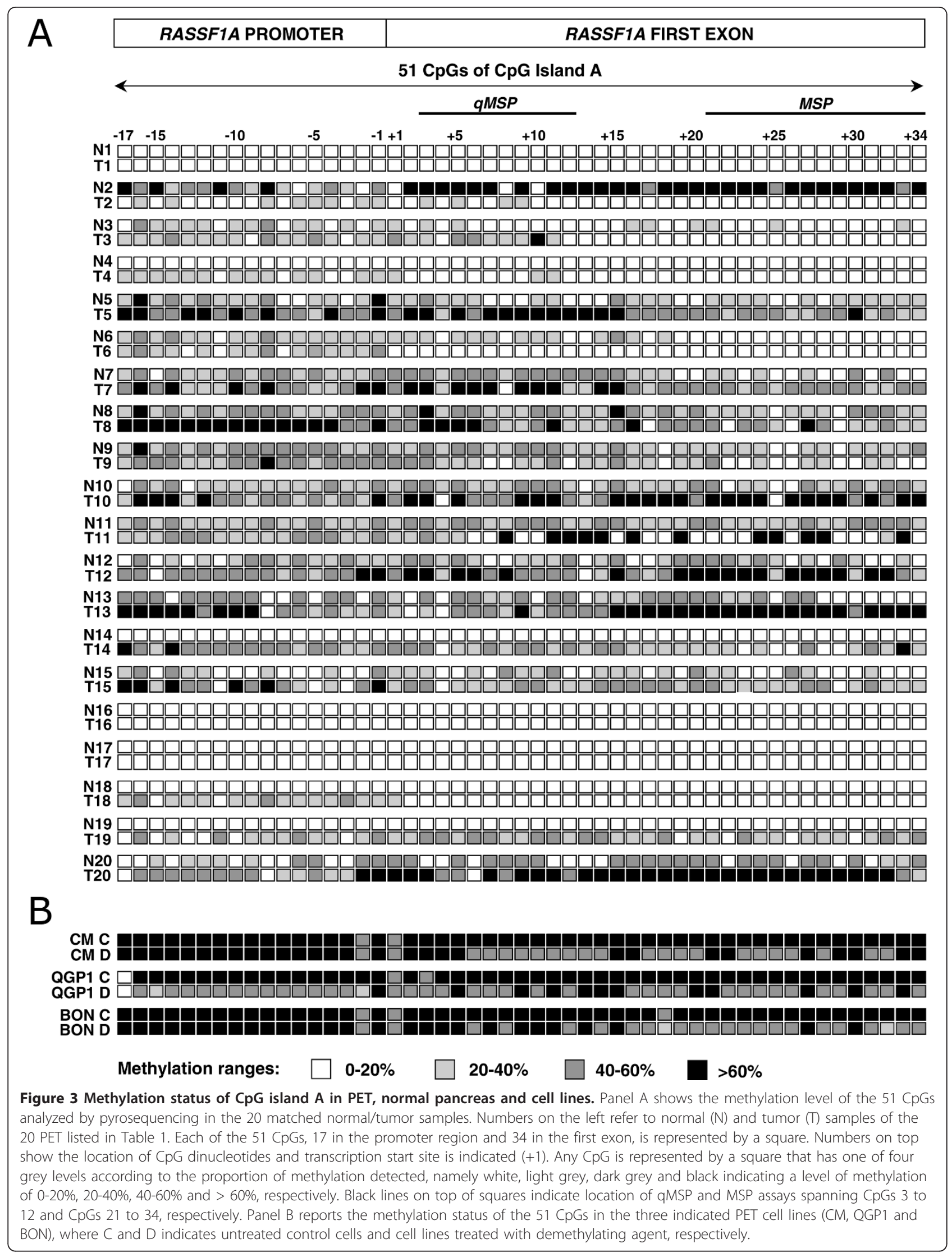


Table 2 Pyrosequencing analysis of 20 matched samples: average methylation values of $51 \mathrm{CpGs}$ in CpG island $A$ of RASSF1A, of which 17 CpGs in the promoter and 34 CpGs in the first exon

\begin{tabular}{|c|c|c|c|}
\hline \multirow{2}{*}{ Cases* } & \multicolumn{3}{|c|}{ Average methylation (\%) } \\
\hline & Promoter & First Exon & CpG island A \\
\hline $\mathrm{N} 1$ & 4 & 2 & 3 \\
\hline $\mathrm{T} 1$ & 2 & 4 & 3 \\
\hline N2 & 34 & 75 & 60 \\
\hline $\mathrm{T} 2$ & 20 & 11 & 14 \\
\hline N3 & 29 & 14 & 19 \\
\hline T3 & 37 & 19 & 25 \\
\hline N4 & 2 & 2 & 2 \\
\hline $\mathrm{T} 4$ & 23 & 8 & 13 \\
\hline N5 & 37 & 22 & 28 \\
\hline T5 & 59 & 56 & 57 \\
\hline N6 & 27 & 18 & 22 \\
\hline T6 & 29 & 10 & 17 \\
\hline N7 & 40 & 35 & 37 \\
\hline $\mathrm{T7}$ & 53 & 52 & 52 \\
\hline N8 & 47 & 37 & 40 \\
\hline T8 & 77 & 46 & 57 \\
\hline N9 & 43 & 32 & 35 \\
\hline T9 & 46 & 29 & 35 \\
\hline N10 & 35 & 34 & 34 \\
\hline T10 & 49 & 72 & 64 \\
\hline N11 & 37 & 38 & 38 \\
\hline T11 & 38 & 43 & 41 \\
\hline N12 & 28 & 18 & 22 \\
\hline $\mathrm{T} 12$ & 42 & 66 & 57 \\
\hline N13 & 25 & 34 & 31 \\
\hline $\mathrm{T} 13$ & 67 & 65 & 66 \\
\hline N14 & 4 & 3 & 4 \\
\hline T14 & 49 & 30 & 37 \\
\hline N15 & 36 & 28 & 31 \\
\hline T15 & 50 & 37 & 41 \\
\hline N16 & 14 & 8 & 10 \\
\hline T16 & 10 & 9 & 9 \\
\hline N17 & 3 & 2 & 2 \\
\hline T17 & 3 & 5 & 4 \\
\hline N18 & 4 & 18 & 13 \\
\hline T18 & 32 & 7 & 16 \\
\hline N19 & 10 & 6 & 8 \\
\hline T19 & 25 & 37 & 33 \\
\hline N20 & 25 & 36 & 32 \\
\hline T20 & 43 & 80 & 67 \\
\hline
\end{tabular}

${ }^{*} \mathrm{~N}=$ normal pancreas, $\mathrm{T}=\mathrm{PET}$.

The comparison between mRNA expression levels and methylation status by MSP showed that the two events were not significantly associated (Wilcoxon test, $P=$ 0.07). When comparing RASSF1A expression data with percent of methylation by qMSP, Spearman's test revealed the presence of an inverse correlation $(\mathrm{r}=-0.35$, $P=0.08)$, which became significant when the average methylation of the $51 \mathrm{CpGs}$ of pyrosequencing data was considered ( $\mathrm{r}=-0.57, P=0.01$ ) (Figure $5 \mathrm{~A}$ ). A major contribution to this latter association was provided by the first exon $(\mathrm{r}=-0.61, P=0.007)$ while methylation of promoter did not correlate significantly $(\mathrm{r}=-0.44, P>0.05)$ (Figure 5B).

We then searched for a role of specific CpGs in the transcription regulation of RASSF1A using pyrosequencing data (Additional file 3, Figure S2). The highest degree of correlation was found for CpG5 (inner graph in Additional file 3, Figure S2). The expression of RASSF $1 A$ inversely correlated with the methylation of this CpG ( $\mathrm{r}=-0.78, P=0.001)$.

RASSF1A expression is present in PET cell lines despite a strong methylation in CpG island $A$ and is enhanced by treatment with demethylating agents

The three PET cell lines CM, QGP1, BON showed a level of methylation above $90 \%$ by qMSP (Additional file 3, Figure S1B). Pyrosequencing analysis confirmed the high level of methylation throughout the CpG island A (Figure 3B). Interestingly, RASSF1A was always expressed at similar level in all the untreated cell lines (Figure 4B). RASSF1A was always expressed in all the untreated cell lines, but at higher level in BON compared to QGP1 and CM.

In order to evaluate the effect of methylation on gene expression of RASSF1A, the three cell lines CM, QGP1 and $\mathrm{BON}$ were treated with the demethylating agent 5'aza-2'-deoxycytidine (DAC). Treatment reduced methylation by about $25 \%$ from the starting level. The main changes of methylation regarded the CpGs included in the first exon (Figure 3B). However, DAC treatment induced a significant increase of RASSF1A expression in QGP1 and CM, but not in BON cell line (Figure 4B). The mRNA expression levels and the average methylation of the $51 \mathrm{CpG}$ within CpG island A showed a significant inverse correlation $(\mathrm{r}=-0.73, P=0.004)$.

Isoform RASSF1C is highly expressed in PET at variance with the other six isoforms generated from RASSF1 locus Seven mRNA isoforms could potentially be expressed by the RASSF 1 locus and four of them (RASSF1-A, D, E and $F$ ) are generated from differential splicing of RASSF1A (Figure 1).

PCR products of RASSF1 isoforms $A, D, E$ and $F$ are resolved by microfluidic chip electrophoresis (Figure 6). RASSF $1 A$ was detected in all PETs while the splicing variants $D, E$ and $F$ were rarely seen: $R A S S F 1 F$ was found in one PET (T7) and two normal pancreas (N3, 




N5), RASSF1D/RASSF1E was expressed in only one sample (N5).

Of the remaining three isoforms, RASSF1G was always absent in PET and normal pancreas, while variant $B$ was always expressed in both normal and PET with no significant difference (data not shown). Conversely, RASSF1C expression level in PETs was significantly higher than that found in normals in 11 of 13 cases $(P$ $=0.001$ ) (Figure $4 \mathrm{C}$ ), having a fold average expression ratio $\mathrm{PET} /$ normal of 11.4 .

\section{CpG island C is never methylated}

Pyrosequencing analysis revealed that none of the 37 CpGs of CpG island C is methylated in PETs and normal pancreas.
Lower expression of RASSF1A and higher expression of RASSF1C in PET with respect to normal pancreas are concomitant events

We tested the relationship between RASSF1A and $R A S S F 1 C$ analyzing the expression ratio $\mathrm{PET} /$ normal. Comparison of RASSF1A PET/normal and RASSF1C PET/ normal expression ratios suggested that the lower expression of the first and the higher expression of the second are two events occurring concomitantly $(\mathrm{r}=0.7, P=0.01)$.

CpG island C is never methylated in PET cell lines and treatment with demethylating agents enhances mRNA expression of RASSF1C

No methylation was detected in $37 \mathrm{CpGs}$ of $\mathrm{CpG}$ island $\mathrm{C}$ in PET cell lines using pyrosequencing and RASSF1C 


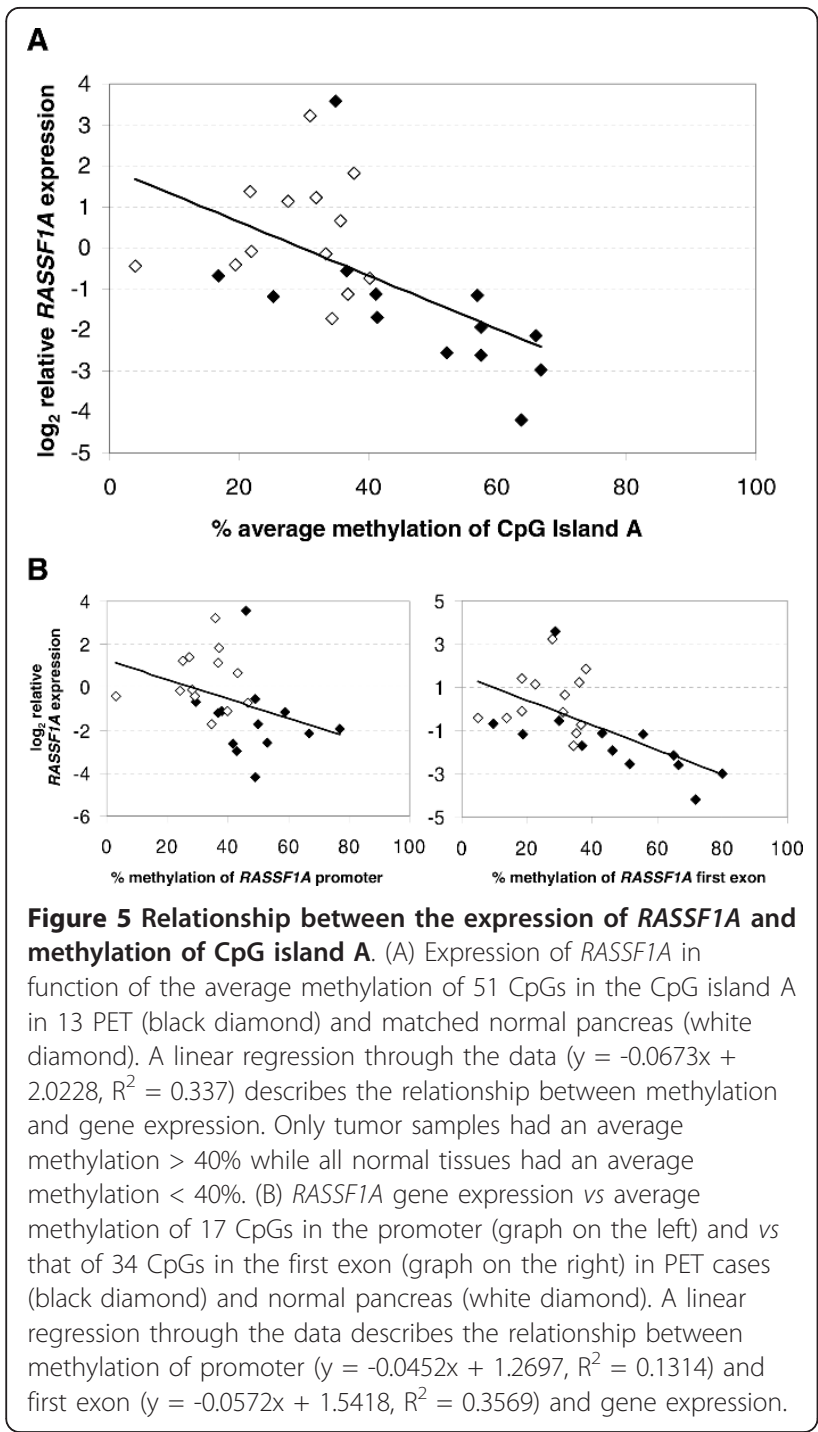

transcript was always found at similar level in untreated cells (Figure 4D). However, treatment of cell lines with DAC upregulated RASSF1C expression significantly in QGP1 and CM, but not in BON cell line.

\section{Discussion}

The suggestion that RASSF1A silencing by methylation is involved in the pathogenesis of PET has been supported by the presence of methylation in the $\mathrm{CpG}$ island A of the gene [1-5]. However, all studies used the same qualitative method to assess methylation and RASSF1A expression has never been analyzed in PET.

In this study we conducted an exhaustive analysis of RASSF1 methylation status in order to define its putative role as a possible tumor specific and transcription regulatory event occurring in PET.

We first analyzed RASSF1 methylation by the qualitative MSP assay because this was the method used in

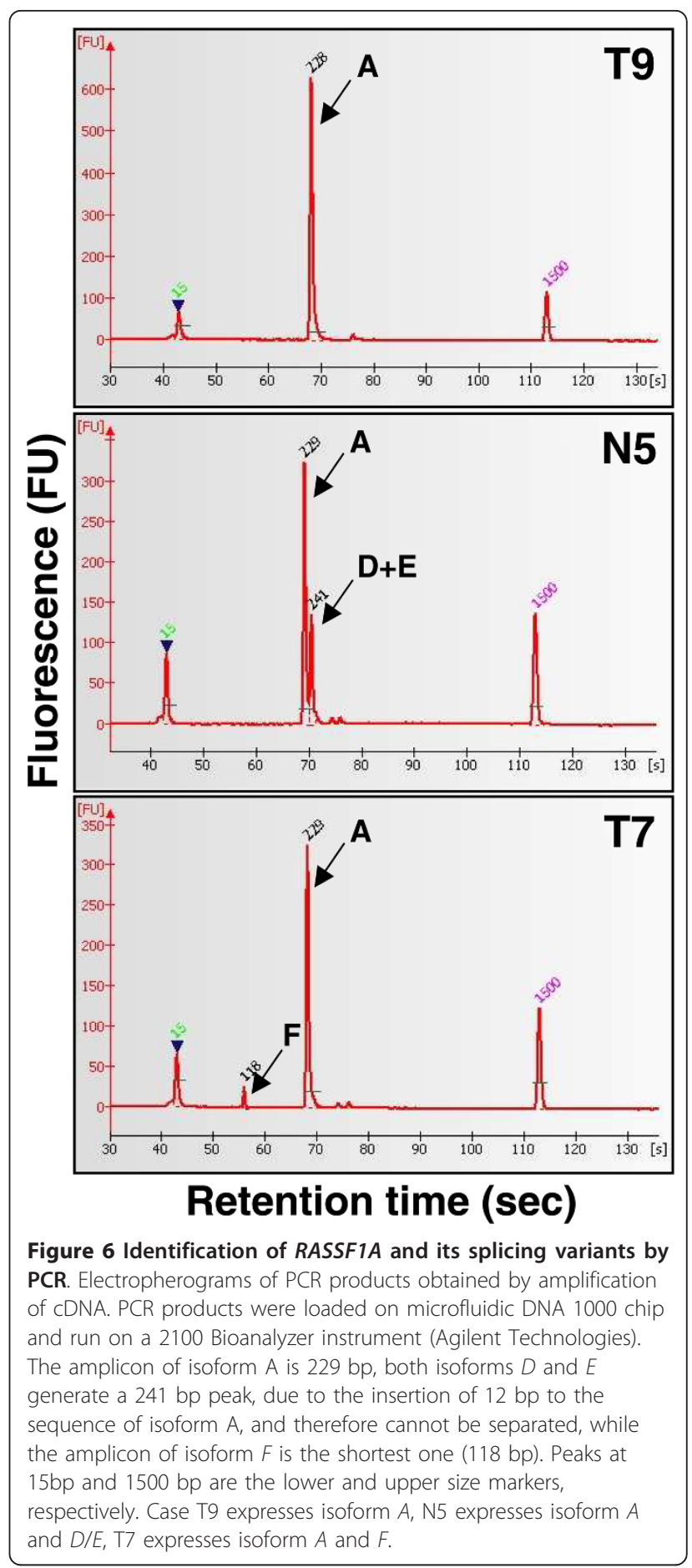

previous studies on PET, and found methylation in $80 \%$ of cases, a frequency similar to that reported in those studies [1-5]. However, MSP is highly sensitive recognizing as little as $0.1 \%$ of methylated alleles [31], thus classifying a sample as methylated on the basis of a minimal proportion of methylated target. We then performed qMSP that has been previously used for the evaluation 
of methylation status of RASSF1A in tumors other than pancreatic $[6-8,10]$. This assay analyzes a region of $C p G$ island $\mathrm{A}$ that has never been investigated in PET. By this quantitative assay, all PET showed some degree of methylation but only $55 \%$ of cases had at least $20 \%$ of their alleles methylated.

In normal pancreas, RASSF1 methylation was found in $65 \%$ of cases by MSP, a value that fit within the range of published studies that used the same technique $[2,4,5]$. By qMSP assay, $45 \%$ of normals had at least $20 \%$ of their alleles methylated.

Finally, we obtained a detailed mapping of RASSF1A methylation by pyrosequencing, assessing the level of methylation of each of 51 CpGs within CpG island A, also encompassing those explored by MSP and qMSP approaches. Pyrosequencing provided a portrait of the complexity of the methylation pattern of tumor cells, where RASSF1A methylation showed a high variability in terms of distribution and level within and among samples. Similar to pancreas, a very complex distribution of methylation of RASSF1A was found in breast cancer [19].

Our pyrosequencing data showed that most of normal and tumor samples had an average methylation levels of the CpG island A below $40 \%$, with the exception of one normal (case 2) and seven tumors, having values above $40 \%$ (Table 2). To classify samples as "methylated", we applied the same cut-off used for qMSP (an average methylation level higher than 20\%). Methylated samples were 13/20 (65\%) in PET and 12/20 (60\%) in normal pancreas. In matched samples, the average methylation level of the $\mathrm{CpG}$ island A was higher in PET than in normal samples in $15 / 20(75 \%)$ cases $(P=0.01)$. Among the 3 normal samples with higher degree of methylation than the neoplastic counterpart, case 2 showed the largest difference of average methylation level between PET and normal. We repeated the pyrosequencing analysis of this case with same results and did not find any particular features in the clinical profile of the patient to associate to the abnormal methylation data.

Although the overall $\mathrm{CpG}$ island A methylation level revealed by pyrosequencing was higher in PET than in normal tissue (Figure 3), normal pancreas displayed considerable methylation levels. The common occurrence of methylation of RASSF1A in normal pancreas suggests that this epigenetic event might represent a "field defect", consisting in widespread epigenetic changes arising early in the pancreas before tumor onset, a hypothesis previously suggested [32,33].

When comparing the three techniques employed in this study to investigate the methylation status, MSP showed different results from those obtained by both qMSP and pyrosequencing. This is consistent with the qualitative (MSP) and quantitative (qMSP and pyrosequencing) nature of these approaches. Conversely, qMSP and pyrosequencing gave comparable results (correlation $r=0.78$ ), thus supporting the choice of using the same cut-off for both quantitative results to classify a sample as methylated.

Whatever the method used to detect methylation, the majority of cases showed concordantly methylated or unmethylated tumor/normal pairs, and, when discordant, the methylation was higher in tumor in most cases, except case 2 (Table 2). This raises the question of whether methylation affects the expression of RASSF1 gene. Indeed, none of the previous papers suggesting the inactivation of RASSF1A in PET due to hypermethylation analyzed gene expression $[2,4,5]$. In the present work, we evaluated the mRNA expression of RASSF1 variants and showed that: i) all PETs and their matched normal tissues expressed $R A S S F 1 A$; ii) the average expression of RASSF1A in PET was 6.8 times lower than that in normal tissues (see Figure 4A and Additional file 2, Table S2); iii) the overall extent of RASSF1A methylation in PET correlated inversely with its expression and the role of methylation of the first exon seems more important than that of the promoter region (see Figure 5). Accordingly, a correlation between RASSF1A expression and the average methylation of the 51 CpGs of island A was found in two of the three PET cell lines analyzed. In all untreated cell lines RASSF1A was always expressed despite a strong methylation in $\mathrm{CpG}$ island $\mathrm{A}$; in particular, BON cell line showed a higher level of RASSF1A expression compared to QGP1 and CM. Moreover, the treatment with the demethylating agent 5'-aza-2'-deoxycytidine enhanced significantly RASSF1A expression in QGP1 and CM, but not in BON. This difference in expression levels and response to demethylating treatment is consistent with a possibly different genetic background. Indeed, it has been recently shown that CM, QGP1, and BON harboured different gene mutations; in particular, they had mutations in FLT1/ VEGFR1, FGFR3, and PIK3CA, respectively [34].

The expression of RASSF1A splicing isoforms $D, E, F$ and $G$, and that of the major variants deriving from alternative promoter usage, RASSF1B and RASSF1C, has never been studied in PET. Here we report that RASSF1 isoforms $D, E, F$ were rarely expressed in PET and normal pancreas; RASSF1G was never found; RASSF1B was always expressed in both PET and normal pancreas, with no significant difference; RASSF1C expression was averagely 11.4 times higher in PET than in normal tissue. Pyrosequencing analysis revealed that all the CpGs within $\mathrm{CpG}$ island $\mathrm{C}$ lacked methylation in both tumor and normal tissues. The same situation was found in PET cell lines, where CpG island $\mathrm{C}$ was never methylated, and RASSF1C was always expressed. Interestingly, treatment with 5 '-aza- 
2'-deoxycytidine enhanced mRNA expression significantly in two of the three cell lines.

The finding of hyperexpression of RASSF1C in PET is of great interest in the light of the recently reported role of RASSF1C in inhibiting $\beta$-catenin degradation [35]. Thus, RASSF1C overexpression may represent a pathogenetic event in PET contributing in sustaining Wnt signaling that has been recently shown to regulate proliferation of pancreatic B-cells [36]. Moreover, RASSF1C has also been implicated in promoting cell migration and attenuating apoptosis in breast cancer cells [37].

\section{Conclusions}

RASSF1A gene is frequently methylated in PET and at higher level than in normal pancreas. However, as no more than $75 \%$ of cases show RASSF1A to be more methylated in PET than normal pancreas, RASSF1 gene methylation cannot be considered a marker lesion for this neoplasm.

RASSF1A is always expressed in PET and normal pancreas and its levels are inversely correlated with gene methylation status.

Isoform RASSF1C is highly expressed in PET at variance with the other six isoforms generated from RASSF1 locus; this suggesting that RASSF1C might play a pathogenetic role in tumor development, in the light of the recent demonstration of its involvement in the regulation of Wnt pathway.

\section{Additional material}

Additional file 1: Additional methods. The file completes the description of methods and experimental conditions employed to obtain the data. The description is subdivided in: quantitative methylationspecific PCR (qMSP), PCR conditions used to amplify samples for DNA pyrosequencing, quantitative reverse-transcription polymerase chain reaction ( $q R T-P C R)$ and immunofluorescence procedure.

Additional file 2: Additional tables. Table S1. Oligonucleotides and experimental conditions used for quantitative MSP (qMSP), DNA pyrosequencing, microfluidic chip-electrophoretic separation (RT-PCR) and quantitative RT-PCR (qRT-PCR). The table lists the primers sequences and the PCR conditions used in GMSP, RT-PCR and qRT-PCR. Table S2. Expression and statistics data of RASSFIA and RASSF1C in PET and normal pancreas obtained by quantitative RT-PCR (qRT-PCR). The table provides the expression data of RASSFIA and RASSFIC and the statistical analysis.

Additional file 3: Additional figures. Figure S1. Analysis of methylation of RASSF1A by methylation-specific PCR (MSP) and quantitative MSP (qMSP). The figure shows examples of MSP results and a graph representing data obtained by qMSP. Figure S2. Pearson's correlations ( $r$ ) between expression of RASSF1A and the average methylation of single CpGs. The graph shows the Pearson's correlation values ( $r$ ) between RASSFIA expression level and the average methylation level for each $\mathrm{CpG}$ of the $\mathrm{CpG}$ island $\mathrm{A}$.

\section{Acknowledgements}

This work was supported by Associazione Italiana Ricerca Cancro (AIRC); Fondazione CariParo, Padova, Italy; Fondazione Cariverona, Verona, Italy; Ministero della Salute e Ministero dell'Università, Roma, Italy.

\section{Author details}

'Department of Pathology and Diagnostics, University of Verona, Verona, Italy. ${ }^{2}$ ARC-NET Center for Applied Research on Cancer, the Hospital and University of Verona, Verona, Italy. ${ }^{3}$ Department of Pathology and Laboratory Medicine, National Cancer Institute and University of Milan, Milan, Italy. ${ }^{4}$ Department of Surgery and Oncology, the Hospital and University of Verona, Verona, Italy.

\section{Authors' contributions}

GM and EA designed and carried out the experiments, analyzed the results, performed the statistical analysis and wrote the manuscript. MD performed cell cultures and demethylating treatments. VD and CF performed part of the experiments. LB selected the series based on clinical data and tissue availability. GP and MF evaluated critically the data and assisted in manuscript preparation. AS conceived the study and finalized the manuscript. All authors read and approved the final manuscript.

\section{Competing interests}

The authors declare that they have no competing interests.

Received: 31 January 2011 Accepted: 12 August 2011

Published: 12 August 2011

\section{References}

1. Arnold CN, Sosnowski A, Schmitt-Graff A, Arnold R, Blum HE: Analysis of molecular pathways in sporadic neuroendocrine tumors of the gastroentero-pancreatic system. Int J Cancer 2007, 120(10):2157-2164.

2. Dammann $R$, Schagdarsurengin U, Liu L, Otto N, Gimm O, Dralle $H$, Boehm BO, Pfeifer GP, Hoang-Vu C: Frequent RASSF1A promoter hypermethylation and K-ras mutations in pancreatic carcinoma. Oncogene 2003, 22(24):3806-3812.

3. House MG, Herman JG, Guo MZ, Hooker CM, Schulick RD, Lillemoe KD, Cameron JL, Hruban RH, Maitra A, Yeo CJ: Aberrant hypermethylation of tumor suppressor genes in pancreatic endocrine neoplasms. Ann Surg 2003, 238(3):423-431, discussion 431-422.

4. Liu L, Broaddus RR, Yao JC, Xie S, White JA, Wu TT, Hamilton SR, Rashid A: Epigenetic alterations in neuroendocrine tumors: methylation of RASassociation domain family 1 , isoform $A$ and 16 genes are associated with metastasis. Mod Pathol 2005, 18(12):1632-1640.

5. Pizzi S, Azzoni C, Bottarelli L, Campanini N, D’Adda T, Pasquali C, Rossi G, Rindi G, Bordi C: RASSF1A promoter methylation and 3p21.3 loss of heterozygosity are features of foregut, but not midgut and hindgut, malignant endocrine tumours. J Pathol 2005, 206(4):409-416.

6. Gonzalgo ML, Yegnasubramanian S, Yan G, Rogers CG, Nicol TL, Nelson WG, Pavlovich CP: Molecular profiling and classification of sporadic renal cell carcinoma by quantitative methylation analysis. Clin Cancer Res 2004, 10(21):7276-7283.

7. Lehmann U, Berg-Ribbe I, Wingen LU, Brakensiek K, Becker T, Klempnauer J, Schlegelberger B, Kreipe H, Flemming P: Distinct methylation patterns of benign and malignant liver tumors revealed by quantitative methylation profiling. Clin Cancer Res 2005, 11(10):3654-3660.

8. Lehmann U, Langer F, Feist H, Glockner S, Hasemeier B, Kreipe H: Quantitative assessment of promoter hypermethylation during breast cancer development. Am J Pathol 2002, 160(2):605-612.

9. van der Weyden L, Adams DJ: The Ras-association domain family (RASSF) members and their role in human tumourigenesis. Biochim Biophys Acta 2007, 1776(1):58-85.

10. Xing M, Cohen Y, Mambo E, Tallini G, Udelsman R, Ladenson PW, Sidransky D: Early occurrence of RASSF1A hypermethylation and its mutual exclusion with BRAF mutation in thyroid tumorigenesis. Cancer Res 2004, 64(5):1664-1668.

11. Agathanggelou A, Honorio S, Macartney DP, Martinez A, Dallol A, Rader J, Fullwood P, Chauhan A, Walker R, Shaw JA, et al: Methylation associated inactivation of RASSF1A from region 3p21.3 in lung, breast and ovarian tumours. Oncogene 2001, 20(12):1509-1518.

12. Benbrahim-Tallaa L, Waterland RA, Dill AL, Webber MM, Waalkes MP: Tumor suppressor gene inactivation during cadmium-induced malignant transformation of human prostate cells correlates with overexpression of de novo DNA methyltransferase. Environ Health Perspect 2007 115(10):1454-1459. 
13. Geli J, Kiss N, Lanner F, Foukakis T, Natalishvili N, Larsson O, Kogner P, Hoog A, Clark GJ, Ekstrom TJ, et al: The Ras effectors NORE1A and RASSF1A are frequently inactivated in pheochromocytoma and abdominal paraganglioma. Endocr Relat Cancer 2007, 14(1):125-134

14. Greenspan EJ, Jablonski MA, Rajan TV, Levine J, Belinsky GS, Rosenberg DW: Epigenetic alterations in RASSF1A in human aberrant crypt foci. Carcinogenesis 2006, 27(7):1316-1322.

15. Li Y, Wei Q, Cao F, Cao X: Expression and promoter methylation of the RASSF1A gene in sporadic breast cancers in Chinese women. Oncol Rep 2008, 19(5):1149-1153.

16. Liu L, Yoon JH, Dammann R, Pfeifer GP: Frequent hypermethylation of the RASSF1A gene in prostate cancer. Oncogene 2002, 21(44):6835-6840.

17. Lo KW, Kwong J, Hui AB, Chan SY, To KF, Chan AS, Chow LS, Teo PM, Johnson PJ, Huang DP: High frequency of promoter hypermethylation of RASSF1A in nasopharyngeal carcinoma. Cancer Res 2001, 61(10):3877-3881.

18. Qian ZR, Sano T, Yoshimoto K, Yamada S, Ishizuka A, Mizusawa N, Horiguchi H, Hirokawa M, Asa SL: Inactivation of RASSF1A tumo suppressor gene by aberrant promoter hypermethylation in human pituitary adenomas. Lab Invest 2005, 85(4):464-473.

19. Yan PS, Shi H, Rahmatpanah F, Hsiau TH, Hsiau AH, Leu YW, Liu JC, Huang TH: Differential distribution of DNA methylation within the RASSF1A CpG island in breast cancer. Cancer Res 2003, 63(19):6178-6186

20. Dammann R, Li C, Yoon JH, Chin PL, Bates S, Pfeifer GP: Epigenetic inactivation of a RAS association domain family protein from the lung tumour suppressor locus 3p21.3. Nat Genet 2000, 25(3):315-319.

21. Rong $R$, Jin W, Zhang J, Sheikh MS, Huang $Y$ : Tumor suppressor RASSF1A is a microtubule-binding protein that stabilizes microtubules and induces G2/M arrest. Oncogene 2004, 23(50):8216-8230.

22. Shivakumar L, Minna J, Sakamaki T, Pestell R, White MA: The RASSF1A tumor suppressor blocks cell cycle progression and inhibits cyclin D1 accumulation. Mol Cell Biol 2002, 22(12):4309-4318.

23. Vos MD, Martinez A, Elam C, Dallol A, Taylor BJ, Latif F, Clark GJ: A role for the RASSF1A tumor suppressor in the regulation of tubulin polymerization and genomic stability. Cancer Res 2004, 64(12):4244-4250.

24. Agathanggelou A, Cooper WN, Latif F: Role of the Ras-association domain family 1 tumor suppressor gene in human cancers. Cancer Res 2005, 65(9):3497-3508.

25. Liu L, Guo C, Dammann R, Tommasi S, Pfeifer GP: RASSF1A interacts with and activates the mitotic kinase Aurora-A. Oncogene 2008, 27(47):6175-6186.

26. Liu L, Tommasi S, Lee DH, Dammann R, Pfeifer GP: Control of microtubule stability by the RASSF1A tumor suppressor. Oncogene 2003, 22(50):8125-8136.

27. Heitz PU, Komminoth P, Perren A: Tumors of the endocrine pancreas. Lyon, France: IARC Press; 2004.

28. Di Gioia S, Bianchi P, Destro A, Grizzi F, Malesci A, Laghi L, Levrero M, Morabito A, Roncalli M: Quantitative evaluation of RASSF1A methylation in the non-lesional, regenerative and neoplastic liver. BMC Cancer 2006, 6:89.

29. Colella S, Shen L, Baggerly KA, Issa JP, Krahe R: Sensitive and quantitative universal Pyrosequencing methylation analysis of $\mathrm{CpG}$ sites. Biotechniques 2003, 35(1):146-150.

30. Waki T, Tamura G, Sato M, Motoyama T: Age-related methylation of tumor suppressor and tumor-related genes: an analysis of autopsy samples. Oncogene 2003, 22(26):4128-4133.

31. Herman JG, Graff JR, Myohanen S, Nelkin BD, Baylin SB: Methylationspecific PCR: a novel PCR assay for methylation status of CpG islands. Proc Natl Acad Sci USA 1996, 93(18):9821-9826.

32. Ahuja N, Issa JP: Aging, methylation and cancer. Histol Histopathol 2000, 15(3):835-842.

33. Guo M, House MG, Hooker C, Han Y, Heath E, Gabrielson E, Yang SC, Baylin SB, Herman JG, Brock MV: Promoter hypermethylation of resected bronchial margins: a field defect of changes? Clin Cancer Res 2004, 10(15):5131-5136.

34. Corbo V, Beghelli S, Bersani S, Antonello D, Talamini G, Brunelli M, Capelli P, Falconi M, Scarpa A: Pancreatic endocrine tumours: mutational and immunohistochemical survey of protein kinases reveals alterations in targetable kinases in cancer cell lines and rare primaries. Ann Oncol 2011.
35. Estrabaud E, Lassot I, Blot G, Le Rouzic E, Tanchou V, Quemeneur E, Daviet L, Margottin-Goguet F, Benarous R: RASSF1C, an isoform of the tumor suppressor RASSF1A, promotes the accumulation of beta-catenin by interacting with betaTrCP. Cancer Res 2007, 67(3):1054-1061.

36. Rulifson IC, Karnik SK, Heiser PW, ten Berge D, Chen H, Gu X, Taketo MM, Nusse R, Hebrok M, Kim SK: Wnt signaling regulates pancreatic beta cell proliferation. Proc Natl Acad Sci USA 2007, 104(15):6247-6252.

37. Reeves ME, Baldwin SW, Baldwin ML, Chen ST, Moretz JM, Aragon RJ, Li X, Strong DD, Mohan S, Amaar YG: Ras-association domain family $1 \mathrm{C}$ protein promotes breast cancer cell migration and attenuates apoptosis. BMC Cancer 10:562

\section{Pre-publication history}

The pre-publication history for this paper can be accessed here: http://www.biomedcentral.com/1471-2407/11/351/prepub

doi:10.1186/1471-2407-11-351

Cite this article as: Malpeli et al:: Methylation-associated downregulation of RASSF1A and up-regulation of RASSF1C in pancreatic endocrine tumors. BMC Cancer 2011 11:351.

\section{Submit your next manuscript to BioMed Central and take full advantage of:}

- Convenient online submission

- Thorough peer review

- No space constraints or color figure charges

- Immediate publication on acceptance

- Inclusion in PubMed, CAS, Scopus and Google Scholar

- Research which is freely available for redistribution

Submit your manuscript at www.biomedcentral.com/submit
C) Biomed Central 